\title{
ANÁLISIS POR ESPECTROSCOPIA \\ DE INFRARROJOS (FTIR) DEL EXUDADO \\ DEL ÁRBOL DE ALGARROBO Prosopis juliflora (FABACEAE)
}

\section{INFRARED SPECTROSCOPY (FTIR) ANALISYS OF THE CAROB TREE EXUDATE Prosopis juliflora (FABACEAE)}

Felipe Gallegos P. ${ }^{1}$, Yolanda López F. ${ }^{2}$ \& Lorena Meneses $\mathbf{O}^{1 *}$

Recibido: 5 de mayo 2020 / Aceptado: 30 de junio 2020

Publicado en línea: 2 de julio de 2020

DOI: $10.26807 /$ ia.v8i2.168

Palabras claves: Acacia senegal, algarrobo, FTIR, goma de algarrobo, goma arábiga, Prosopis juliflora.

Keywords: Acacia senegal, arabic gum, carob tree, carob tree gum, FTIR, Prosopis juliflora.

\section{RESUMEN}

La goma de algarrobo es un exudado proveniente de las ramas y troncos de los árboles del género Prosopis, tiene una semejanza a la goma arábiga y posee potenciales aplicaciones en el área farmacéutica, de alimentos, industria cos-

1 Pontificia Universidad Católica del Ecuador, Facultad de Ciencias Naturales y Exactas, Escuela de Ciencias Químicas, Quito, Ecuador (edfelipe28@gmail.com; *correspondencia: Immeneses@ puce.edu.ec).

2 Centro de Investigación en Alimentación y Desarrollo A.C, Hermosillo, Sonora, México (lopezf@ciad.mx) 
mética, etc. El algarrobo se encuentra en regiones secas y semi secas del Ecuador. En el presente trabajo se analizaron por espectroscopia FTIR, tres muestras de goma de algarrobo y una muestra de goma arábiga grado alimenticio, para la obtención y comparación de espectros. Las muestras provenientes del árbol Prosopis juliflora, fueron recolectadas en tres áreas de la provincia de ManabíEcuador: Bahía de Caráquez (Zona 1), Portoviejo (Zona 2) y Puerto López (Zona 3). Se obtuvieron espectros FTIR de cada una de las muestras y se compararon con espectros de la bibliografía. Los espectros de infrarrojos presentaron semejanza entre los dos tipos de gomas, algarrobo y arábiga, así como con los de la bibliografía; se efectuó una comparación analítica entre los dos tipos para la obtención de porcentajes de correlación, estos fueron de 80,37; 79,83 y 79,66 $\%$ para las zonas 1, 2 y 3 respectivamente. Los resultados obtenidos abren una línea de investigación para la posible utilización de la goma de algarrobo ecuatoriana como una nueva y accesible alternativa al uso de la goma arábiga.

\section{ABSTRACT}

Carob tree gum is an exudate obtained from the stems and branches of Prosopis trees. It is a natural product with potential applications in food, pharmaceutical, cosmetic industry, etc. It is found in dry and semi dry regions of Ecuador. In the present work, samples originated from Prosopis juliflora tree, collected from three zones of Manabí, province of Ecuador: Bahía de Caráquez (Zone 1), Portoviejo (Zone 2) and Puerto López (Zone 3), and one sample of arabic gum, food grade from Acacia senegal tree used as reference, were analyzed by infrared spectroscopy, to obtain and compare the spectrums of the each one of the samples. Infrared spectrums of both gums were obtained and compared with bibliography. The FTIR spectra exhibited a good correlation between the two types of gums and with the literature; an analytical comparison was made to obtain the correlation percentage between both gums. The results were $80.37,79.83$ and $79.66 \%$ for 1,2 and 3 zones, respectively. Thus, a research line is open for the possible use of carob gum, as a new alternative and accessible, instead of arabic gum. 


\section{INTRODUCCIÓN}

Se denomina exudado vegetal a toda aquella sustancia, goma, látex o resina, que es producida y expulsada en estado líquido por una planta ya sea por estrés natural o daños físicos (Goswami y Naik, 2014).

La goma de algarrobo y la goma arábiga se definen como exudaciones gomosas de leguminosas, presentes en áreas áridas y semiáridas. La primera es obtenida a partir de la corteza y ramas del árbol Prosopis juliflora, o especies relacionadas al género Prosopis, procedente de América del Norte. La segunda, a partir de los árboles de Acacia senegal o seyal, originarias de África del Norte (Vernon, Beristain y Pedroza, 2000).

Las gomas naturales cumplen diferentes funciones en diversas áreas de la química. En el área de alimentos se emplea en la fabricación de caramelos y como emulsificante, en la encapsulación de saborizantes y como estabilizante en bebidas, además, en la industria farmacéutica en la elaboración de pastillas, como medio de transporte de algunos fármacos o como aglutinante, entre otras (López, Goycoolea, Wang, 2009).
En las gomas se encuentran compuestos fenólicos, que son sustancias con anillos bencénicos en su estructura. Estos compuestos ayudan al correcto funcionamiento del sistema vegetal y actúan como mecanismos de defensa ante agentes patógenos. Así mismo, el consumo de productos con alto contenido fenólico favorece al cuerpo humano, al actuar como antioxidantes y como parte de la defensa en el sistema inmune. La ingesta diaria reduce la incidencia de enfermedades cardiovasculares, diabetes, cáncer y alergias; los polifenoes actúan como inhibidores de radicales libres en la sangre protegiendo el ADN e inactivando enzimas involucradas en procesos degenerativos (Ozcan, Akpinar, Ersan y Delikanli, 2014).

La goma de algarrobo tiene un alto reconocimiento en México y se conoce como goma de mezquite. Ha contribuido económicamente a muchas comunidades del centro norte de México y sur de Estados Unidos, por lo que actualmente la goma de mezquite ha ganado un alto interés en el uso industrial (Vernon et al., 2000). 
Para el caso ecuatoriano, la comercialización de la goma de algarrobo es nula. En la provincia de Manabí existe un cierto reconocimiento, por los lugareños, de que esta exudación permite combatir afecciones estomacales.

El Ecuador posee amplias regiones áridas y semiáridas ubicadas en su mayoría en la región costa, con una extensión aproximada de 9593,82 km² (Verbist, Santibañez, Gabriels y Soto, 2010). Burghardt, Brizuela, Pía, Albán y Palacios (2010), estudiaron la presencia del género Prosopis en las costas de Ecuador y Perú, haIlando varias especies de Prosopis en Manabí, Guayas y El Oro, y también en la zona de Loja.

El aprovechamiento de estas especies para la obtención de goma de algarrobo y la evaluación de ésta como posible sustituto de la goma arábiga, debe ser puesto en consideración. Lo anterior fomentaría la implementación de bosques de algarrobo en las zonas semiáridas y áridas del país, además del uso de las zonas actuales.

Debido a la semejanza, tanto de estructura química y fisicoquímica, entre la goma arábiga y la goma de algarrobo, ésta última supone una nueva forma de satisfacer la demanda de este tipo de aditivos ante el crecimiento industrial que se vive en los últimos años (Armijos, 2016). En el presente estudio se propone analizar soluciones metanólicas de goma o exudado de algarrobo (Prosopis juliflora) ecuatoriana y comparar con la goma arábiga (Acacia senegal), por medio de espectroscopia de infrarrojos.

\section{MATERIALES Y MÉTODOS}

\section{Preparación de Muestras}

Tres zonas ecuatorianas fueron muestreadas: Bahía de Caráquez (Zona 1), Portoviejo (Zona 2) y Puerto López (Zona 3). La recolección de muestras de goma algarrobo se efectuó según las metodologías propuestas por Hassan (2008), Yebeyen, Lemenih y Feleke (2009), Kauther y Hussien (2018) y Sibaja (2015); para ello se eligieron al azar 20 árboles de cada 
zona que presentaron evidencia física de goma. Las gomas fueron limpiadas, trituradas y almacenadas. Además, se adquirió una muestra de goma arábiga grado alimenticio.

\section{Análisis por espectroscopia IR}

La caracterización por espectroscopia FTIR, fue realizada en los laboratorios de la Pontificia Universidad Católica del Ecuador.

Se llevó a cabo una extracción metanólica de cada una de las muestras de goma, siguiendo las metodologías descritas por Mohammedelnour, et. al (2017).

Se pesaron 5 g de cada una de las muestras de goma de algarrobo triturada y arábiga, en una balanza analítica marca Mettler Toledo ML204. Se disolvieron en $50 \mathrm{~mL}$ de metanol absoluto (Sigma Aldrich). Se maceró por 48 horas a obscuras evitando el contacto con la luz. Las soluciones fueron filtradas a vacío con papel filtro cuantitativo (MN 640 m). Las soluciones resultantes se concentraron a $40{ }^{\circ} \mathrm{C}$ en una estufa, marca Binder ED 115, hasta eliminar completamente el metanol.

Se empleó un equipo de espectroscopia de infrarrojos Perkin Elmer BX, ATR miracle. Mediante el software Sprectrum se leyó el espectro de fondo y seguido se realizaron las lecturas de los extractos de cada una de las muestras de goma. Se obtuvieron los espectros infrarrojos en transmitancia \%T. Finalmente, se transformaron los espectros a unidades de absorbancia (U.A) para una mejor apreciación de bandas y posterior comparación con espectros reportados en la literatura.

\section{RESULTADOS}

En la Tabla 1 se presentan las tres zonas de muestreo seleccionadas para este estudio, así como algunas características geográficas de cada una de ellas. 
Tabla 1. Ubicación de las zonas de muestreo en la provincia de Manabí para la obtención de la goma de algarrobo

\begin{tabular}{|c|c|c|c|c|}
\hline $\mathbf{N}^{\circ}$ & Ubicación & Coordenadas & $\begin{array}{l}\text { Rango de } \\
\text { Temperatura } \\
\text { (junio-julio) }\end{array}$ & $\begin{array}{l}\text { Promedio } \\
\text { de lluvia } \\
\text { (junio-julio) }\end{array}$ \\
\hline Zona 1 & $\begin{array}{l}\text { Bahía de Caráquez, } \\
\text { San Vicente. }\end{array}$ & $\begin{array}{c}\text { Latitud:0³6' 16.45"S } \\
\text { Longitud:80²3'58.71"O }\end{array}$ & $23,0-30,0^{\circ} \mathrm{C}$ & $0,0-1,1 \mathrm{~mm}$ \\
\hline Zona 2 & $\begin{array}{l}\text { Portoviejo, } \\
\text { Jardín Botánico de la } \\
\text { Universidad Técnica } \\
\text { de Portoviejo }\end{array}$ & 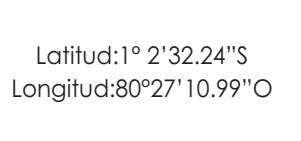 & $17,0-32,0^{\circ} \mathrm{C}$ & $0,0-0,4$ \\
\hline Zona 3 & $\begin{array}{l}\text { Puerto López, Bosque } \\
\text { seco de Algarrobo }\end{array}$ & $\begin{array}{l}\text { Latitud:1³1'52.61"S } \\
\text { Longitud: } 80^{\circ} 44^{\prime} 32.41^{\prime \prime O}\end{array}$ & $21,0-31,0^{\circ} \mathrm{C}$ & $0,0-0,5 \mathrm{~mm}$ \\
\hline
\end{tabular}

Se programaron las fechas de recolección, en la provincia de Manabí, entre los meses junio y julio del 2018. Cabe recalcar que las edades de los árboles de la zona 1 y zona 2 fueron similares, de 5 a 10 años, mientras que la zona 3 contiene árboles de edades jóvenes de 3 a 5 años; esto con el objetivo de observar diferencias de composición entre las gomas (Inamhi, 2018).

\section{Análisis por espectroscopia IR}

Las Figuras 1 a 4 muestran los espectros de infrarrojo obtenidos a partir del análisis de los extractos de tres muestras de goma de algarrobo y de goma arábiga. Cada una de ellas está representada por absorbancia versus el número de onda $\left(\mathrm{cm}^{-1}\right)$, en los cuales se identifican las bandas más significativas. 


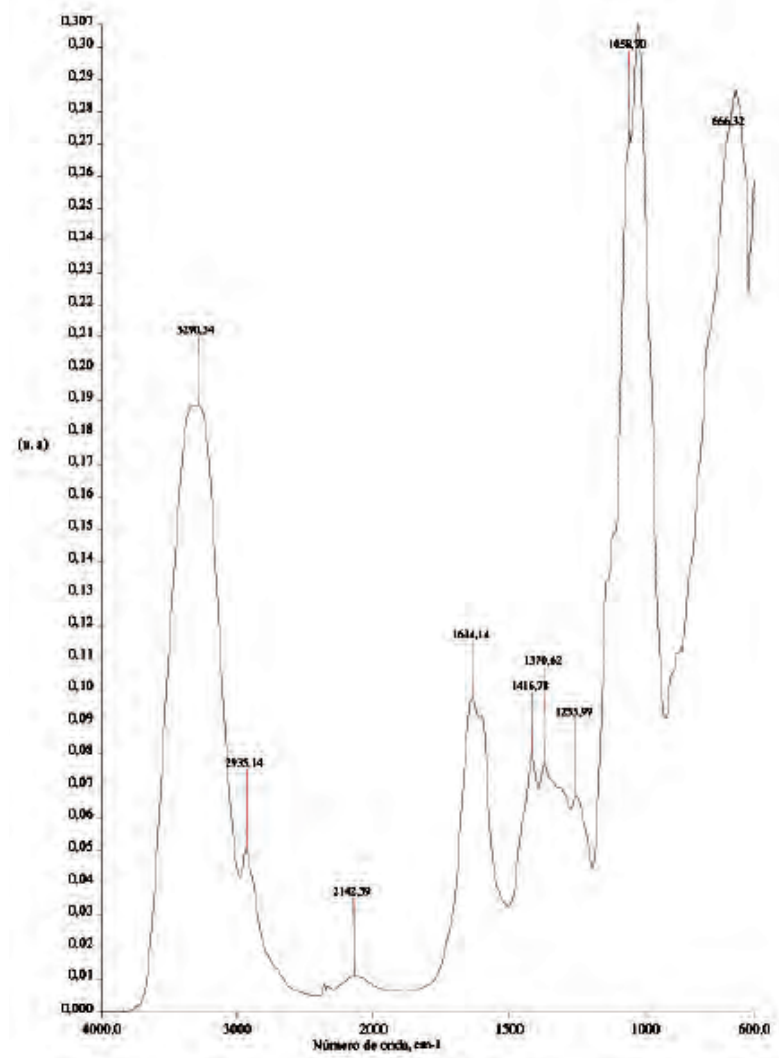

Figura 1. Espectro infrarrojo, absorbancia versus longitud de onda, para la goma de algarrobo de la zona 1, Bahía de Caráquez 
InfoANALÍTICA 8(2)

Julio 2020

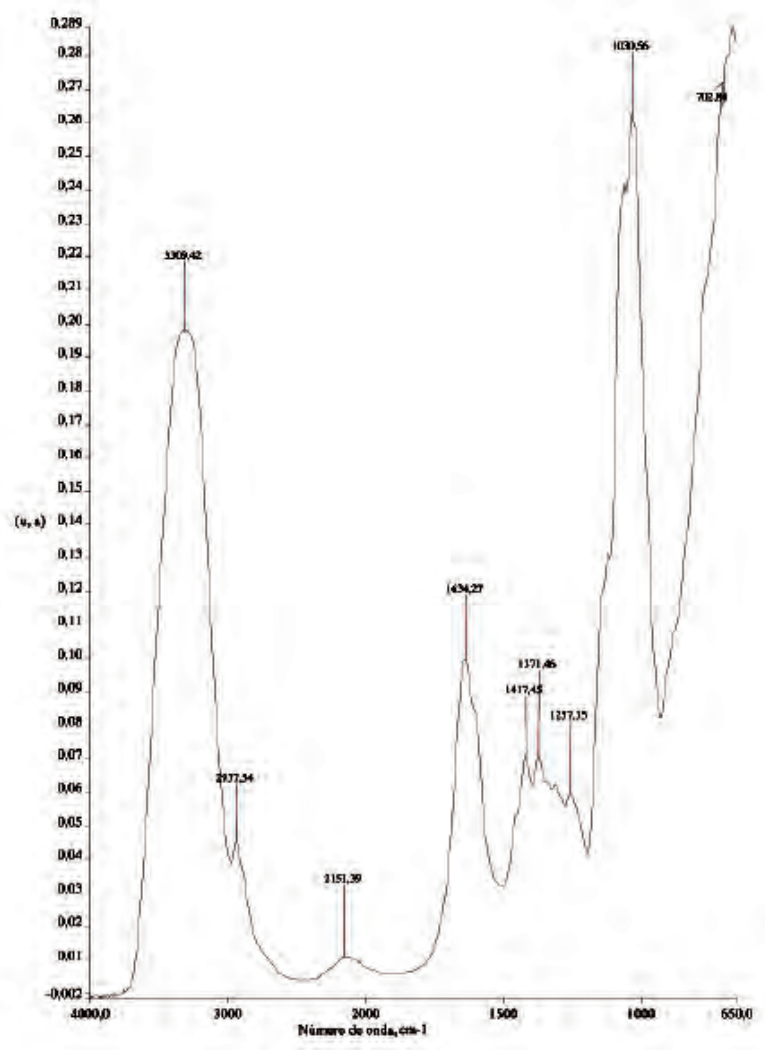

Figura 2. Espectro infrarrojo, para la goma de algarrobo de la zona 2,

Portoviejo 


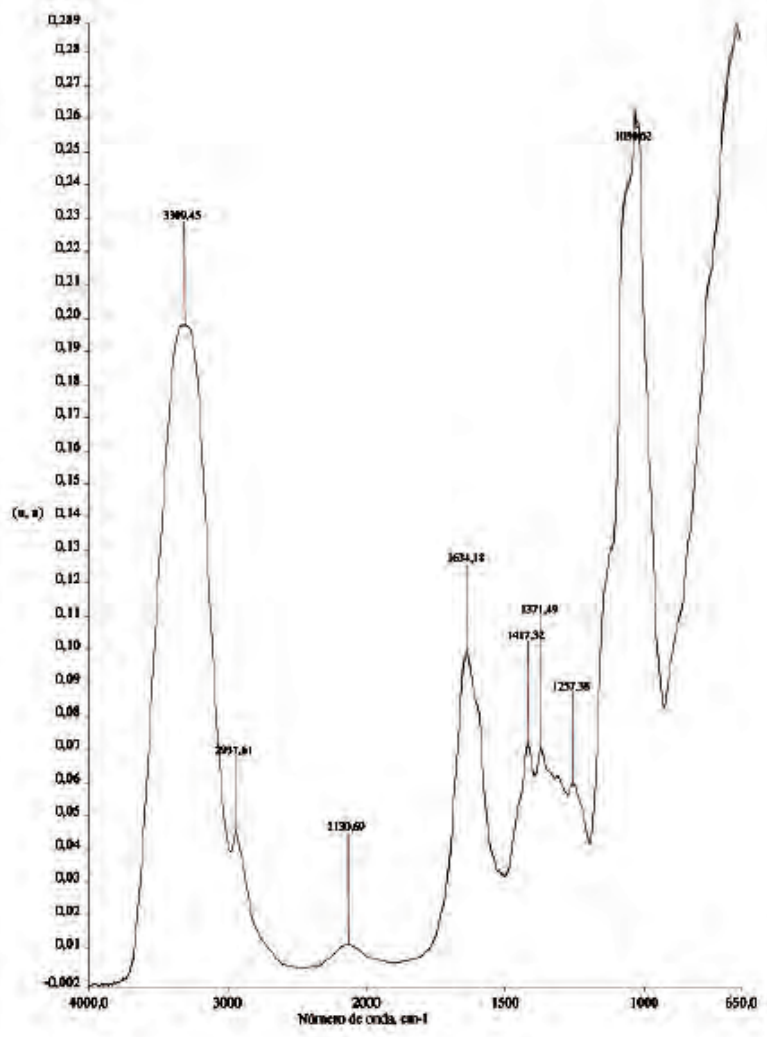

Figura 3. Espectro infrarrojo, para la goma de algarrobo zona 3 , Puerto López 
InfoANALÍTICA 8(2)

Julio 2020

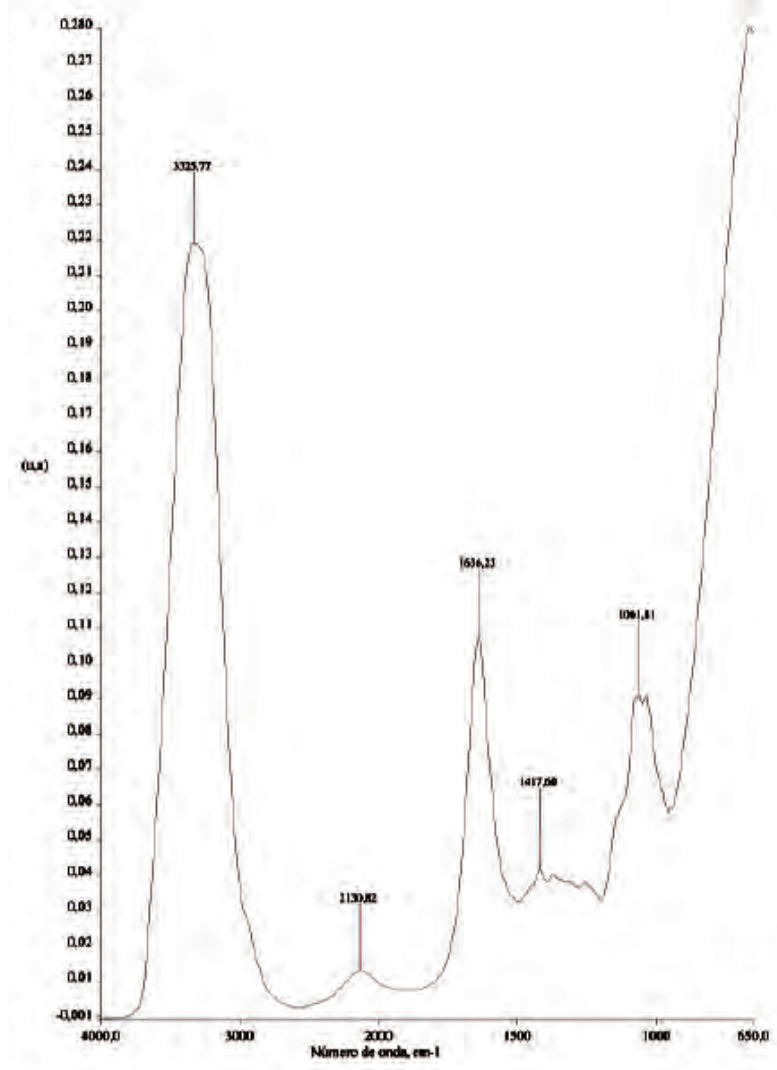

Figura 4. Espectro infrarrojo, para la goma arábiga grado alimenticio 


\section{DISCUSIÓN}

En el presente trabajo se analizaron los compuestos fenólicos encontrados en los extractos de goma arábiga y algarrobo. Entre los picos sobresalientes y relacionados en bibliografía, están los grupos fenólicos a $3210 \sim 3320$ $\mathrm{cm}^{-1}$ (Vasile, Romero, Judis y Mazzobre, 2016). Otra banda característica es la del grupo de los alcanos, en este caso polímeros de carbohidratos, con frecuencias de $2939 \sim 2946 \mathrm{~cm}^{-1}$ encontrado en todas las gomas de algarrobo, menos en la muestra de goma arábiga (López, Córdova, Goycoolea, Valdez, Onofre y Mendoza, 2012). Esto se debe a la presencia de moléculas de agua en la mezcla, lo que usualmente genera una expansión de la señal del grupo O-H solapando otras absorciones de importancia (Palencia, 2018). La aplicación de la segunda derivada ayuda a la separación de picos solapados, sin embargo al ser un procedimiento matemático este posee limitaciones, lo cual podría resultar en una distorsión del espectro (Rieppo, Saarakkala, Närhi, Helminen, Jurvelin y Rieppo. 2012). Los picos 1629 a $1663 \mathrm{~cm}^{-1}$ demuestran la presencia de flavonoides y grupos carbonilo (Vasile et al., 2016). Los picos entre los 1300 y los $1400 \mathrm{~cm}^{-1}$ también son equiparables con bibliografía, representando a los enlaces CC para aromáticos y alcanos (Udo, Odoemelam y Okon, 2017). La banda a $1014 \mathrm{~cm}^{-1}$, indica estiramientos en enlaces C-O (Bashir y Haripriya, 2016).

Bashir y Haripriya, (2016) señalan que los picos a $1400 \mathrm{~cm}^{-1}$ son debido a ácidos urónicos y residuos de ácidos carboxílicos; de igual manera establecen que los picos correspondientes a $1014 \mathrm{~cm}^{-1}$ podrían ser descritos como moléculas de arabinogalactanas.

La comparación analítica de espectros, entre los tres extractos de las muestras de goma de algarrobo contra el de la muestra de goma arábiga comercial, presentó un porcentaje de correlación de 80,37 \% para la goma de la zona 1, 79,83 \% para la goma de la zona 2 y 79,66 \% para la goma de la zona 3. La baja de resolución de los picos y la falta del pico entre $2939 \sim 2946 \mathrm{~cm}^{-1}$ en la goma arábiga generaron una disminución en el porcentaje de correlación. De igual 
forma, los espectros entre la goma de algarrobo joven, zona 3, y la goma de algarrobo madura, zonas 1 y 2, fueron similares, por lo que se puede decir que su estructura química es semejante. De este modo, la composición orgánica no varía con el tiempo de vida del árbol, si no que varía la cantidad de agua y minerales con la cual es exudada, los cuales son aprovechados para el crecimiento del árbol.

La buena correlación entre los espectros de los extractos de goma arábiga y goma de algarrobo, demuestran una estructura química y de composición similares. De igual manera, al comparar los espectros de goma de algarrobo con trabajos relacionados, se puede encontrar una similitud tanto para goma arábiga como para goma de algarrobo (López et al., 2012; Vasile et al., 2016; Elbadawi, Alsabah, y Abuelhassan, 2018).
Por otro lado, se debe mencionar que las disoluciones acuosas de goma de algarrobo presentaron una coloración más oscura que la de goma arábiga. La coloración está relacionada con la cantidad de taninos condensados en las gomas, los cuales provienen de las proantocianidinas. A mayor intensidad, mayor el contenido (Sibaja, 2015). Del mismo modo el trabajo propuesto por Vasile, Romero, Judis, Mattalloni, Virgolini, y Mazzobre, (2019) demostraron un alto contenido de taninos respecto a la goma arábiga. Las proantocianidinas son compuestos fenólicos o polímeros de flavonoides encontrados en gran variedad de plantas. Son las responsables de otorgar propiedades antioxidantes y consecuentemente una coloración café-rojiza (IBQ, 2014). En base a este estudio, se deduce que la goma de algarrobo posee un mayor contenido fenólico en relación a la goma arábiga.

\section{CONCLUSIÓN}

El estudio por espectroscopia de infrarrojos demostró que los espectros de las tres muestras de goma de algarrobo y de la goma arábiga grado alimenticio son comparables, por lo que comparten una estructura química similar. Los porcentajes de correlación demuestran un valor de alrededor del $80 \%$, considerado relativamente elevado. 
La variación de edad de los árboles, entre 3 a 10 años, no influye en la estructura química de la goma de algarrobo, los espectros de las gomas de las zonas 1, 2 y 3 fueron semejantes entre ellos.

De igual forma los espectros y la identificación de picos, obtenidos experimentalmente, fueron comparables con espectros encontrados en bibliografía para la goma de algarrobo y goma arábiga.
Los resultados obtenidos representan una línea base para el desarrollo de conocimiento en relación a las posibles aplicaciones de la goma de algarrobo, como alternativa de uso de la goma arábiga comercial. Esto generaría beneficio para la comunidad ecuatoriana, además de fomentar el aprovechamiento de los recursos que existen en las regiones áridas y semiáridas del país.

\section{LISTA DE REFERENCIAS}

Armijos, S. (2016). Bebidas. El sector se inclina a lo natural. Vistazo, Proyectos Especiales Industrias. Recuperado de: http://anfab.com/wp/wp-content/uploads/2016/08/SUPLEMENTO-REVISTA-VISTAZO-agosto-2016-386.pdf

Bashir, M. y Haripriya, S. (2016). Assessment of physical and structural characteristics of almond gum. International Journal of Biological Macromolecules. 93, 476-482.

Burghardt, A., Brizuela, M., Pía, M., Albán, L. y Palacios, R. (2010). Análisis numérico de las especies de Prosopis L. (Fabaceae) de las costas de Perú y Ecuador. Revista Peruana de Biología. 17(3), 317-323.

Elbadawi, A., Alsabah, Y. y Abuelhassan, H. (2018). Physical and Electrical Properties of Gum Arabic. En A. Abdalbasit (Ed.). Gum Arabic: Structure, Properties, Application and Economics (pp. 75-89). London: Academic Press. Elsevier. 
Goswami, S. y Naik, S. (2014). Natural gums and its pharmaceutical application. Journal of Scientific and Innovative Research. 3(1), 112-121.

Hassan, M. (2008). Comparison of the Physiochemical Properties of Gum Arabic (Acacia Senegal L.) Produced in Sandy and Gardude soils in Sudan. (Tesis de Maestria). Universidad de Gezira. Egipto.

Instituto Nacional de Meteorología e Hidrología Ecuador, Inamhi. (2018). Anuarios Meteorológicos. Inamhi. Recuperado de: http://www.serviciometeorologico.gob.ec/ docum_institucion/anuarios/meteorologicos/

Instituto Químico Biológico, IBQ. (2014). Proantocianidinas. IBQ España. Recuperado de: http://www.iqb.es/monografia/fichas/proantocianina.htm

Kauther, A. y Hussien, D. (2018). Physicochemical and Funcional Properties of the Gum Arabic from Acacia Senegal. Annals. Food Science and Technology. 19(1), 27-34.

López, Y., Córdova, R., Goycoolea, F., Valdez, M., Onofre, J. y Mendoza, J. (2012). Classification and physicochemical characterization of mesquite gum (Prosopis spp.). Food Hydrocolloids. 26, 159-166.

López, Y., Goycoolea, F. y Wang, W. (2009). Other exudates: tragancanth, karaya, mesquite gum and larchwood arabinogalactan. En G. Phillips y P. Williams (Ed.). Handbook of Hydrocolloids (pp. 495-534) Cambridge: Woodhead Publishing.

Mohammedelnour, A., Mirghani, M., Kabbashi, N., Alam, Z., Musa, H. y Abdullah, A. (2017). Effect of solvent types on phenolics content and antioxidant activities of Acacia polyacantha gum. International Food Research Journal. 24, 369-377.

Ozcan, T., Akpinar, A., Ersan, L. y Delikanli, B. (2014). Phenolics in Human Health. International Journal of Chemical Engineering and Applications. 5(5), 393-396.

Palencia, M. (2018). Functional transformation of Fourier-transform mid-infrared spectrum for improving spectral specificity by simple algorithm based on wavelet-like functions. Journal of Advanced Research. 14, 53-62. 
Rieppo, L., Saarakkala, S., Närhi, T., Helminen, H., Jurvelin, J. y Rieppo, J. (2012). Application of second derivative spectroscopy for increasing molecular specificity of Fourier Transform infrared spectroscopic imaging of articular cartilage. Osteoarthritis Research Society International. 20(5), 451-459.

Sibaja, R. (2015). Propiedades Fisicoquímicas y Funcionales de las Gomas de Acacia cochliacantha y Acacia farnesiana. (Tesis de Doctorado). Instituto Politécnico Nacional. México.

Udo, I., Odoemelam, S. y Okon, E. (2017). Physicochemical and FTIR studies on Acacia senegal and Anacardium occidentale blends. Journal of Industrial and Environmental Chemistry. 1(1), 31-35.

Vasile, F., Romero A., Judis, M. y Mazzobre, M. (2016). Prosopis alba exudate gum as excipient for improving fish oil stability in alginate-chitosan beads. Food Chemistry. 190, 1093-1101.

Vasile, F., Romero, A., Judis, M., Mattalloni, M., Virgolini, M. y Mazzobre, M. (2019). Phenolics composition, antioxidant properties and toxicological assessment of Prosopis alba exudate gum. Food Chemistry. 285, 369-379.

Verbist, K., Santibañez, F., Gabriels, D. y Soto, G. (2010). Atlas de zonas áridas de América Latina y el Caribe. Recuperado de https://unesdoc.unesco.org/ark:/48223/ pf0000216333

Vernon, E., Beristain, C. y Pedroza, R. (2000). Mesquite gum (Prosopis gum). Developments in Food Science. 41, 217-238.

Yebeyen, D., Lemenihb, M. y Feleke, S. (2009). Characteristics and quality of gum arabic from naturally grown Acacia senegal (Linne) Willd. trees in the Central Rift Valley of Ethiopia. Food Hydrocolloids. 23, 175-180. 